\title{
Investigación de la violencia sexual contra hombres (Balance teórico)
}

\author{
Investigation of sexual violence against men \\ (Theoretical balance)
}

https://doi.org/10.15332/iust.v0i17.2423

\section{Daniela López, Gómez}

Historiadora por la Universidad Javeriana y Magíster en Ciencia Política por la Universidad de los Andes. Analista de prensa del Cinep e investigadora del "Grupo de Investigación Red Internacional de Politica Criminal Sistémica Extrema Ratio UN" Universidad Nacional de Colombia.

Correo electrónico: sinelegeun_bog@unal.edu.co

\section{Resumen}

En este artículo se presenta un balance teórico sobre los trabajos que se han realizado a propósito de la violencia sexual contra los hombres, y su objetivo principal es visibilizar los trabajos que hay sobre este tema e impulsar la investigación de este tipo de violencia en Colombia. El balance hace parte de una investigación más extensa sobre violencia sexual contra hombres en el marco del conflicto armado colombiano, y se realizó a partir de aproximadamente 150 trabajos, entre libros y artículos. Ahora bien, esta investigación muestra tres temas fundamentales desde los cuales se ha estudiado la violencia sexual contra los hombres: 1. La violencia sexual y organismos internacionales. 2. Aspectos conceptuales y teóricos sobre este tipo de violencia. 3. Rutas y vías para visibilizar y tratar esta problemática, temáticas que sin duda alguna son importantes para la comprensión del tema.

Palabras clave: Violencia sexual; género; mancha de la homosexualidad; masculinidad hegemónica; feminización; sesgo anti gay.

\section{Abstract}

This article to present a theoretical balance on the work that has been done on sexual violence against men and its main objective is to make visible the work on this topic and to promote the investigation of this type of violence in Colombia. The report is part of a more extensive investigation on sexual violence against men in the context of the Colombian armed conflict, and was carried out from approximately 150 works, including books and articles. Now, this work shows three fundamental issues from which sexual violence against men has been studied. 1. Sexual violence and international organizations. 2. Conceptual and theoretical aspects of this type of violence. 3. Routes and ways to visualize and treat this problem, topics that are undoubtedly important for the understanding of the subject.

Keywords: Sexual violence; gender; stain of homosexuality; hegemonic masculinity; feminization; anti gay bias. 


\section{Résumé}

Cet article vise à présenter un équilibre théorique sur le travail qui a été fait sur le thème de la violence sexuelle contre les hommes et son objectif principal est de rendre visible le travail sur ce sujet et de promouvoir l'enquête sur ce type de violence en Colombie. Le solde fait partie d'une enquête plus approfondie sur les violences sexuelles contre les hommes dans le contexte du conflit armé colombien et a été réalisée à partir d'environ 150 ouvrages, dont des livres et des articles. Maintenant, ce travail montre trois thèmes fondamentaux à partir desquels la violence sexuelle contre les hommes a été étudiée. 1. violence sexuelle et organisations internationales: 2 . aspects conceptuels et théoriques de ce type de violence; 3 . Itinéraires et moyens de rendre visible et de résoudre ce problème, questions qui sont sans aucun doute importantes pour la compréhension du sujet.

Mots-clés: Violence sexuelle; le sexe; tache d'homosexualité; masculinité hégémonique; féminisation; anti biais gay. 


\section{Investigación de la violencia sexual contra hombres ${ }^{1}$ (Balance teórico)}

Daniela López Gómez

\section{INTRODUCCIÓN}

Actualmente en escenarios nacionales e internacionales, los temas relacionados con la violencia sexual han cobrado gran importancia, sobre todo cuando las víctimas han sido las mujeres. Esto se debe a un proceso histórico de grandes luchas, que no empezó, de entrada, con el reconocimiento de este tipo de violencia. El primer logro fue el reconocimiento de las mujeres y su voz propia en la sociedad, y los siguientes estuvieron relacionados con el espacio, tanto privado como público (Pérez, 2011, pp. $140-144)^{2}$.

Posterior al reconocimiento de la violencia sexual, este tipo de violencia ha ido desembocando en la creación de políticas a nivel mundial, cuya intención es prevenir y tratar a las víctimas de este hecho y constituirlo en una grave violación a los derechos humanos, a los derechos de las mujeres y niños y, por ende, a un delito que por su gravedad es considerado crimen de lesa humanidad.

Ello se ha reflejado en los postulados del Convenio de Ginebra y sus protocolos adicionales, el Estatuto de Roma y los informes de la ONU, entre otros. También en las continuas investigaciones que narran las experiencias de las mujeres, y documentan diferentes estrategias para tratar este problema y luchar para que esto no vuelva a suceder.

No obstante, la violencia sexual reconocida hoy como uno de los principales hechos victimizantes de las mujeres, ha dejado de lado al hombre como posible víctima de este hecho. Es así como el campo de la violencia sexual se ha convertido en un escenario exclusivo de las mujeres $\mathrm{y}$, por ende, la legislación, las políticas

1 Artículo de investigación desarrollado por el grupo de Investigación Escuela de Derecho Penal Nullum Crimen Sine Lege UN, reconocido y clasificado en Colciencias 2017.

2 Aparece entonces la primera y segunda ola del feminismo. La primera, referida a la política legal, educativa e igualdad de condiciones; la segunda, de 1960-1990, relacionada con otros derechos como los reproductivos, dentro de los cuales se encontraba el problema de la violencia sexual y la violencia doméstica. Momentos históricos que dieron importantes cambios respecto a la mujer en la sociedad y a la opresión sexista contra la mujer.

De esta manera, el feminismo inició, no solo una nueva etapa histórica en donde las mujeres entraban a jugar un punto importante, sino que centró a la mujer como víctima sobreviviente, contrario a estudios y enfoques anteriores en donde se centraba únicamente en la víctima. De aquí sobresalen diferentes corrientes, tales como el feminismo liberal, el feminismo radical, el feminismo socialista, entre otros. 
nacionales e internacionales y los estudios e investigaciones se han limitado a cubrir la figura de la mujer y en algunos casos las de los niños, sin embargo, al hombre se le ha fortalecido aquella figura de inmunidad para este tipo de hechos victimizantes. Él sigue siendo una figura que no puede ser víctima de la violencia sexual, como bien lo explica la profesora Lara Stemple (2009), se evidencia un tipo de jerarquización de las víctimas, donde se olvida que el hombre no es un ser monolítico (Stemple, 2009, p. 635). Sumado a esto, se crea la idea del cuerpo del hombre, en términos de Ruth Graham (2006, p. 196), como un cuerpo inviolable e impenetrable. Ello lo demuestran las pocas investigaciones que se han desarrollado al respecto tanto a nivel nacional (Colombia) como internacional.

Por esta razón, el presente artículo está dirigido a realizar un breve pero detallado balance teórico que muestra la mayoría de estudios, o por lo menos los más importantes a propósito de esta problemática, con el objetivo de brindar un documento que promueva, facilite, invite y ayude a desarrollar este tipo de investigaciones en Colombia. En este artículo se pretende dejar claros algunos conceptos importantes para comprender la problemática de la violencia sexual contra el hombre, algunos debates en torno a este tema, certezas que ya se tienen en cuanto a la existencia de estos hechos y reflexiones que posibilitan el abordaje de este tipo de estudios.

Este propósito desemboca en otras intenciones, las cuales se refieren a, primero, invitar a la investigación de esta problemática; segundo, llamar la atención sobre la existencia de estos hechos contra los hombres, para abrir un nuevo tema que resulte en la construcción de políticas y estrategias que faciliten las narraciones de víctimas masculinas, con el objetivo de diseñar soluciones y tratamientos para ellos; tercero, contribuir a los estudios de género, en el sentido de seguir promoviendo una igualdad, en vez de seguir reproduciendo la idea de la víctima mujer - pasiva y susceptible a tipos de violencia única como la sexual, reconozca que no solo a ella por ser "mujer" se viola y abusa, sino que al hombre, "macho", también se le vulnera su más íntimo espacio. Es decir, que ellos también han sido y lo son, cuerpos violentados para demostrar poder, dominio, humillación y triunfo del victimario ${ }^{3}$.

Ahora bien, en este artículo se mostrarán, en su mayoría, estudios y trabajos producidos por académicos extranjeros, y se hará alusión a muy pocos estudios producidos en América Latina y, específicamente, en Colombia, porque la producción en torno a esta problemática ha sido mucho más fructífera en otros países, sobre todo en Estados Unidos, donde han estudiado el problema de la violencia sexual del hombre desde diferentes escenarios, tales como la prisión; la guerra; el hogar, entre otros. Sin embargo, estos estudios aún siguen siendo escasos y en todos, por lo general, reclaman la poca atención a esta problemática y sus evidentes consecuencias en la sociedad.

3 Ruth Graham (2006, p. 201), en su análisis recomienda que es necesario construir el cuerpo masculino como violable y susceptible de daño. Es definitivo el abandono de la reducción del hombre al ser agresor únicamente y analizar entonces cómo se entiende la vulnerabilidad de este. 
Por otra parte, este balance que se pretende mostrar a continuación es fruto de una investigación mayor sobre la violencia sexual contra los hombres en el marco del conflicto armado colombiano y es uno de los productos de la investigación. Se han realizado, por medio de la sistematización, alrededor de 150 trabajos sobre violencia sexual contra el hombre, entre artículos (todos publicados en revistas académicas) y algunos libros. A lo largo de este escrito se hará alusión a algunas páginas web de gran interés y a algunos documentales que han representado un valioso aporte para la comprensión del tema.

Aunque la cifra da cuenta de la poca producción del tema, también evidencia que las reflexiones han ido creciendo y que las preguntas sobre la violencia sexual contra los hombres están cobrando cada vez más importancia. Esto se puede ver en el número de publicaciones sobre el tema que se ha producido a partir del año 2000. Finalmente, se mencionará un estudio realizado en Colombia, pues es el único del cual se tiene reporte, sin embargo, todo el balance teórico que se presenta está en función de adquirir herramientas teóricas para analizar este tipo de problemáticas en Colombia y en América Latina.

Cabe aclarar que aun cuando se intenta hacer alusión a la mayoría de estudios que hay sobre violencia sexual contra hombres, quedan faltando muchos importantes, que seguramente han debido mencionarse, pero el límite del escrito no lo ha permitido, por lo cual, se hace necesario, sintetizar lo mejor posible y reunir más que los estudios y sus autores, las principales tendencias, posturas teóricas y debates que giran en torno a este tema.

\section{LA VIOLENCIA SEXUAL CONTRA EL HOMBRE}

El tema de violencia sexual ha sido ampliamente explorado, sin embargo, el hombre ha sido dejado de lado, por lo menos como víctima. Es así como, por lo general, se le define siempre como el victimario de este tipo de violencia. Ello tiene fundamentos fuertes y certeros, ya que, en su mayoría, la población víctima de este tipo de violencia ha sido femenina y los perpetradores han sido los hombres. No obstante, ello ha influido en que no se reconozca; por parte de las políticas públicas, organizaciones internacionales y nacionales, ni por medios de comunicación, el problema de los hombres abusados sexualmente en distintos escenarios, ya sean domésticos, carcelarios o de violencia política.

En cuanto a la violencia sexual, esta se consideraba, en tiempos anteriores, como un atentado hacia el honor, es decir que, si una mujer era violada o agredida sexualmente, la ofensa no recaía sobre ella, sino sobre su esposo o sobre su comunidad, era considerado como un acto contra la propiedad, o un crimen relacionado con la disciplina de la tropa (Lewis, 2009, p. 20). Esta postura se ha modificado desde diversos escenarios, tales como organismos internacionales, medios de 
comunicación, academia y salud, entre otros. Una muestra de ello es el cambio de noción de violencia sexual por parte del derecho internacional, como explica Dustin Lewis, quien identifica hoy en día, dos tipos de delitos en torno a la agresión de violencia sexual: 1. La agresión a la integridad física o mental de un individuo y, 2. La agresión a la autonomía sexual de una persona (Lewis, 2009, p. 20).

Uno de los hechos que influyeron en este cambio de noción fueron las cartas de Londres y de Tokio, en las cuales empezó una cierta visibilización del tema, aun cuando no se mencionaba la violencia sexual como tal, pero por lo menos se empezó a hacer referencia a "malos tratos" en el que de una u otra manera la violencia sexual estaba incluida (Lewis, 2009, p. 36).

Posteriormente, la violencia sexual se empezó a hacer mucho más evidente por medio del IV Acuerdo de Ginebra en 1949, Título III, sección I, artículo 27 y todos sus protocolos adicionales (Protocolo adicional I, 1977 y Protocolo adicional II, 1977) en los cuales se incluyó un apartado mucho más claro sobre tratos degradantes y se prohibió definitivamente la violación, sobre todo en contra de la mujer. Este cambio fue motivado en gran parte por los demandes que se evidenciaron después de la Segunda Guerra Mundial, tanto por parte de Alemania como de Rusia, lo cual mostró la situación que afrontaban las mujeres durante la guerra.

Ya para los años 90 del siglo XX, fruto de los abusos y desmandes producidos, tanto en el conflicto de ex Yugoslavia como en el de Ruanda, se crearon tribunales internacionales, los cuales marcaron un paso fundamental para el reconocimiento de la violencia sexual, pues las cifras de este delito fueron significativas e impactantes (Zarkov, 2001; Ruvinsky, 2012; Freivogel, 2010). Sin embargo, todos estos reconocimientos siguieron enfocándose principalmente en la mujer (Ruvinsky, 2012; Lewis, 2009).

Años después, en 1998 el Estatuto de Roma de la Corte Penal Internacional ${ }^{5}$ reconoció la violencia sexual y la definió como crimen de lesa humanidad. Igualmente, reconoció todo delito con motivo de género. Este Estatuto a diferencia de los artículos consignados en los Convenios de Ginebra, exceptuando el protocolo II del Convenio ${ }^{6}$, definen la violencia sexual sin reducirla a la mujer, sino que contempla una posición mucho más general. Menciona claramente la problemática de género, pero no se reduce a un grupo específicamente como se muestra a continuación en el artículo 7 (Huertas y Amaya, 2016):

4 Comité Internacional de la Cruz Roja. https://www.icrc.org/spa/resources/documents/misc/ protocolo-i.htm

5 El texto del Estatuto de Roma que se distribuyó como documento A/CONF.183/9, de 17 de julio de 1998, enmendado por los procèsverbaux de 10 de noviembre de 1998, 12 de julio de 1999, 30 de noviembre de 1999, 8 de mayo de 2000, 17 de enero de 2001 y 16 de enero de 2002. El Estatuto entró en vigor el 1o de julio de 2002. http://www.un.org/spanish/law/icc/statute/spanish/rome_statute(s). pdf.

6 Comité Internacional de la Cruz Roja. https:/www.icrc.org/spa/resources/documents/misc/ protocolo-i.htm 
Se entenderá por "crimen de lesa humanidad" cualquiera de los actos siguientes cuando se cometa como parte de un ataque generalizado o sistemático contra una población civil y con conocimiento de dicho ataque... Violación, esclavitud sexual, prostitución forzada, embarazo forzado, esterilización forzada o cualquier otra forma de violencia sexual de gravedad comparable...

... Cometer atentados contra la dignidad personal, especialmente los tratos humillantes y degradantes...

... xxii) Cometer actos de violación, esclavitud sexual, prostitución forzada, embarazo forzado, definido en el apartado f) del párrafo 2 del artículo 7, esterilización forzada y cualquier otra forma de violencia sexual que también constituya una infracción grave de los Convenios de Ginebra...

... Adoptará medidas adecuadas para asegurar la eficacia de la investigación y el enjuiciamiento de los crímenes de la competencia de la Corte. A esos efectos, respetará los intereses y las circunstancias personales de víctimas y testigos, entre otros la edad, el género, definido en el párrafo 3 del artículo 7, y la salud, y tendrá en cuenta la naturaleza de los crímenes, en particular los de violencia sexual, violencia por razones de género y violencia contra los niños... (Estatuto de Roma de la Corte Penal Internacional, artículo 8, Crímenes de Guerra).

Adicionalmente, se debe considerar la importancia del Protocolo de Estambul, Manual para la investigación y documentación eficaces de la tortura y otros tratos o penas crueles, inhumanos o degradantes, emitido en el 2014 por Naciones Unidas, y en donde reconocen dentro de la tortura: La Violencia sexual sobre los genitales, vejaciones, introducción de instrumentos, violación (Naciones Unidas, Protocolo de Estambul, G. Examen de los métodos de tortura, p. 56). Este documento, al igual que el Estatuto de Roma, considera la violencia sexual de forma general y no especifica si es mujer u hombre, lo cual es un gran avance respecto a los demás documentos.

Lo anterior evidencia un logro en términos del reconocimiento de la violencia sexual a nivel internacional, con efectos muy fuertes a nivel nacional, que han llevado a reconsiderar una serie de políticas y legislaciones en torno a la violencia de género y a la posición de la mujer tanto en situaciones de paz como de conflicto. Sin embargo, los vacíos sobre la problemática del hombre son evidentes, pues como puede verse en los diferentes decretos y protocolos, si bien la violencia de género se reconoce, la mención al hombre es casi nula, de tal manera que se reproduce el mensaje de que la población sujeta a protección especial son las mujeres y los niños, asumiendo así que el hombre no sufre este tipo de violencia.

Ahora bien, el escenario internacional no es el único que presenta vacíos respecto a esta problemática; la academia, uno de los escenarios por excelencia para visibilizar, concientizar y reflexionar sobre diversos problemas, deja ver un gran descuido sobre los hombres y este tipo de hechos. 
Por lo general, los análisis que se han hecho sobre la violencia sexual contra el hombre provienen de la criminalística, algunos del derecho y pocos de la ciencia política. Trabajos que han adoptado conceptos y enfoques del feminismo y de las nuevas masculinidades, aun cuando estos dos últimos estudian muy poco este tipo de situaciones respecto a los hombres.

\section{BALANCE}

Para empezar, es necesario analizar el punto de partida de todos los estudios sobre violencia sexual contra hombres, el cual es la preocupación por la invisibilización de esta problemática y la pregunta del porqué se ha ignorado. Por otro lado, casi todos coinciden en la realización de una reflexión sobre el feminismo, su importancia en la sociedad y su incidencia en el olvido de la violencia sexual contra hombres.

Los trabajos que acá se retoman, tienen muchas coincidencias aun cuando guardan un enfoque particular, sin embargo, los puntos de encuentro son inevitables y casi que puede asegurarse que existe un consenso en cuanto a las causas de la violencia sexual contra hombres, la razón del olvido de este tema desde distintos escenarios y las posibles soluciones para ello.

Del balance que se ha realizado, se pueden destacar varios temas: 1. La atención de la violencia sexual contra hombres por parte de organismos internacionales; 2. Las definiciones de violencia sexual, las causas y consecuencias de este tipo de hechos en los hombres y, 3. Las vías para darle visibilidad y tratamiento a la problemática. Cabe aclarar que, se ha preferido agruparlos por temas y no por tipos de textos o enfoques, ya que casi todos los autores que aquí se citan mantienen un orden similar, es decir: 1. Enuncian la invisiblización de la violación contra hombres por parte de los organismos internacionales; 2. Explican y analizar la problemática como tal y, 3. Proponen algunas vías para solucionar este vació temático. Sin embargo, se encuentran algunos estudios que han realizado análisis enfocados a temas particulares, ya sean estos la masculinidad hegemónica, los mitos de la violación contra hombres, la mancha homosexual, entre otros, a los cuales también se hará alusión por su gran aporte en esta temática.

\section{La violencia sexual contra hombres y los organismos internacionales}

Respecto al tema de la invisibilización de la violencia sexual contra los hombres por parte de los organismos internaciones, puede decirse que casi todos los estudios sobre el tema dedican un parte de su análisis a ello, debido a que llama la atención la extensa mención al delito de violencia sexual, sus tratamientos y rutas de prevención, casi siempre enfocadas en las mujeres, mientras que los hombres parecen no ser parte de este tipo de hechos victimizantes, a pesar de contar con la seguridad de que se han presentado varios casos en diversos conflictos a nivel mundial. Este vacío ha 
despertado gran interés y se ha constituido en un escenario que, desde los estudiosos del tema, puede ser definitivo para visibilizar y tratar la violencia sexual contra el hombre.

En primer lugar, están los estudios que se dedican a proponer algunas críticas al espacio jurídico internacional sobre la violación del hombre en contextos de violencia; este el caso de Dustin Lewis (2009) con su trabajo Unrecognized victims: sexual violence against men in conflict settings under international law, quien analiza, cómo desde el campo jurídico del Derecho Internacional Humanitario y del Derecho Penal Internacional, se ha invisibilizado la violencia sexual de los hombres, lo cual ha tenido graves repercusiones, puesto que los países han naturalizado e ignorado este tipo de hechos. Lewis insiste en que categorías como género, sexo y homosexualidad, contribuyen a dicha invisibilización, razón por la cual propone otras formas de entender las situaciones de violencia sexual, tanto de la mujer como del hombre, para darle mayor cabida a este último.

Lewis asegura que el Derecho Internacional Humanitario da por hecho que la violencia de género es igual a la violencia contra la mujer, y esto sucede de igual forma tanto en la Convención contra la Tortura como en el Pacto Internacional de Derechos Civiles y Políticos. Para llegar a esta conclusión, el autor, a lo largo del texto, estudia la evolución del tratamiento de la violencia sexual por medio de instrumentos internacionales y reconoce que se han dado grandes avances, sin embargo, en cuanto a los hombres, evidencia: a. Una clasificación y definición estereotipada en el derecho internacional; b. Una falta de vocabulario para la narración de este tipo de experiencias y, c. Una reducción del concepto de violencia sexual (Lewis, 2009, p. 202).

Otro estudio de la misma línea es el propuesto por Hilmi Zawati (2006), La impunidad o inmunidad: violación masculina en tiempos de guerra y la tortura sexual como crimen de lesa humanidad, en el que nuevamente se llama la atención sobre el vacío existente en los entes jurídicos internacionales respecto a la violencia sexual hacia el hombre. Zawati parte de los casos de Bosnia Hersegovina, Croacia e Irak, para analizar las vejaciones de las cuales los hombres han sido víctimas. Su objetivo es hacer un llamado a los entes nacionales e internacionales con el fin de considerar la violencia sexual como un tema primordial en la agenda de discusión, buscando sea tratado con su debida importancia. Zawati, parte del hecho de que la violencia sexual en tiempos de guerra es la máxima humillación que se puede infligir a un ser humano, y debe ser considerado como uno de los más graves crímenes contra la humanidad, evitando sea eclipsado por otros crímenes como a menudo sucede con el hombre víctima de este tipo de violencia.

Muy ligados a los trabajos anteriores, están los análisis cuyo interés se centran en evidenciar la poca atención de los casos de violencia a los hombres desde la ONU. Entre estos trabajos se encuentran, Del dicho al hecho: la ONU y la violencia sexual contra hombres y niños durante conflictos armados, escrito por Sandesh Sivakumaran (2010), quien además de evidenciar los espacios concretos donde el 
hombre ha sido invisibilizado, se dedica a hacer un pequeño recorrido sobre los trabajos académicos que se han realizado del tema, concluyendo nuevamente en la existencia de un silencio sobre este (pp. 16-17).

A lo largo del estudio, Sivakumaran analiza cada uno de los espacios en donde se habla del tema de la $\mathrm{ONU}^{7}$, sin embargo, encuentra que estos debates han mantenido a las mujeres en el centro y a los hombres se les ha destinado un lugar relegado. Por otra parte, identifica tres tendencias a propósito de las respuestas emitidas por la jurisprudencia de los tribunales penales internacionales de la ONU, estos son, 1. La mención de la violencia sexual no caracterizada, cuya referencia más común es la tortura, 2. Mención de la violencia sexual y su caracterización, pero sin consecuencia alguna y, 3. Se caracteriza la violencia sexual y se imponen las penas, no obstante, los hombres están alejados.

También se ubica el análisis de Augusta del Zotto (2002), titulado Hombre-aHombre violencia sexual en tiempos de guerra. Este trabajo, aun cuando tienen aportes en otros campos, también hace un importante análisis de los discursos políticos y las organizaciones no gubernamentales.

Del Zotto se encarga de determinar los factores culturales e institucionales que han influido en los silencios de los hombres sobre los abusos de los cuales han sido víctimas en medio del conflicto armado, en especial, en el que se refiere a BosniaHerzegovina y Kosovo, su espacio de investigación.

$\mathrm{Su}$ estudio en particular es bastante interesante, por tres razones, la primera es que a diferencia de los trabajos anteriores, aquí el factor cultural es fundamental, en tanto influye en la invisibilización o poca atención al hombre como víctima; la segunda, es el análisis de la institucionalidad, no solo como un campo

7 1. En el debate del Consejo de Seguridad que precedió a la aprobación de la Resolución 1820, la representante permanente de Eslovenia ante la ONU manifestó, en nombre de la Unión Europea, que "la violencia sexual y de género es un tema de especial preocupación para las mujeres y las niñas en situaciones de conflicto, aunque ahora sabemos que los hombres y los niños también están expuestos a ese tipo de violencia y a la tortura de índole sexual”. 2. Un año después, durante el debate en el que se examinó el informe del Secretario General acerca de la Resolución 1820, el delegado de México mencionó una "tendencia al uso de la violencia sexual contra los hombres". 3. En un debate de la Asamblea General acerca de la asistencia humanitaria, la delegada de Noruega expresó la preocupación de su país "por la violencia sexual y de género que cada año destruye la vida de miles de mujeres y niñas, y también la de hombres y niños". 4. En un debate similar durante una reunión del Consejo Económico y Social, el alto comisionado asistente de ACNUR hizo un comentario acerca de las noticias sobre la violencia sexual ejercida contra hombres y niños, pero aclaró que eran "menos frecuentes [que los informes sobre mujeres y niñas] por vergüenza o por miedo a la estigmatización". 5. En el marco de la iniciativa interinstitucional "No más violaciones" se ha sostenido que "aunque las mujeres y las niñas son las principales víctimas de las violaciones, los hombres y los niños también pueden serlo". 6. Por su parte, la Organización Mundial de la Salud ha observado que la violencia de hombres contra hombres no recibe suficiente atención. 7. 5 marzo de 2010, N. 877 de la versión original 8. En 2008, la Oficina para la Coordinación de Asuntos Humanitarios de la ONU (OCAH) convocó a un grupo de expertos para que evaluaran la naturaleza, el alcance y los motivos de la violencia sexual contra hombres y niños durante los conflictos armados. El tema estaba considerado como uno de "los dos tópicos prioritarios para la investigación en materia de violencia de género en situaciones de conflicto" (Sivakumaran, 2010, p. 5). 
invisibilizador de algunos temas sino como campo no constructor de vías que proporcionen espacios de expresión a los hombres víctimas de violencia sexual y, la tercera, porque resalta la participación de la clase política en el olvido de este tipo de violencia como una forma de defender los intereses tanto regionales como nacionales (Del Zotto, 2002, p. 40). Tres elementos que ninguno de los anteriores había explorado con detalle.

También es necesario mencionar uno de los trabajos más completos en cuanto al análisis de la problemática de la violencia sexual contra el hombre, este es el realizado por el profesor Charles Carpenter, titulado Recognizing Gender-Based Violence Against Civilian Men and Boys in Conflict Situations, quien se enfoca en los organismos internacionales y el descuido de la problemática, pero centrándose en el análisis del discurso de todos estos organismos, pues asegura que aunque el planteamiento de la mayoría de los discursos internacionales son inclusivos al hablar de género, casi siempre se terminan enfocando en las mujeres y en los niños, aun cuando tienen conocimiento de casos de hombres.

El trabajo de Carpenter es de gran valor, porque se dedica a analizar y comparar diferentes informes emitidos por dichos organismos con el fin de evidenciar cuantas veces o cómo se hace referencia a la problemática de mujeres y a la de los hombres, cuyo resultado fue la gran extensión de escritos y menciones sobre las mujeres y la poca o nula mención sobre los hombres. Según su análisis, con exactitud afirma, por ejemplo, haber encontrado solo escasas menciones sobre la masculinidad en uno de los documentos de investigación (2006, p. 87).

Con base en lo anterior, Carpenter concluye, como la mayoría de los autores mencionados en este balance, que:

Las tendencias del sector de seguridad humanitaria han hecho grandes adelantos respecto a la violencia contra las mujeres, sin embargo, la exclusión de la victimización de hombres y niños tanto del discurso como de la realidad es problemático tanto para su comprensión como para las políticas (2006, p. 37).

A esta misma conclusión llegan Lara Stemple (2009), Anna Philo Gorrrid (2015) y Chris Anderson (quienes igual que los trabajos anteriores, analizan la exclusión de los hombres en los tratamientos internacionales y de la ONU. Esto, según los autores, sucede precisamente por el lenguaje que se refiere a la violencia sexual, el cual es específicamente lenguaje femenino, es decir, un lenguaje excluyente frente al hombre Stemple, (2009, p. 618); Philo Gorrid (2015, pp. 421-422), es así que, la violencia de género está enfocada en las normas sociales que discriminan a las mujeres.

De estos trabajos es de resaltar el de Lara Stemple, ya que tiene dos aportes fundamentales, los cuales son, en primera medida, la búsqueda y exposición de cifras a propósito de hombres víctimas y, en segunda medida, la diferenciación de los distintos escenarios y contextos de la violencia sexual contra el hombre. Stemple entonces aclara que la violencia sexual se da en varios escenarios y sus lógicas son 
diferentes, entre los cuales están, la violencia sexual en la cárcel ${ }^{8}$; la violencia sexual en el conflicto y la violencia sexual contra población infantil. Aquí es de aclarar que, si bien son los escenarios más comunes, habría que preguntarse por la violación en escenarios de delincuencia común, por la violación en escenarios domésticos y por escenarios laborales ${ }^{9}$ y universitarios ${ }^{10}$.

Según Stemple (2009, p. 606) en EE. UU. el 15.2\% de los hombres han experimentado violencia sexual y según los centros para el control y prevención de enfermedades y El Instituto Nacional de Justicia se reportan 92.700 hombres violados. Según un análisis mundial, el 3\% de los hombres han sido violados, según la Organización Mundial de la Salud entre el 5\% y 10\% de los hombres han sido violados especialmente en países subdesarrollados como Perú, con un $20 \%$. Namibia con un 3,6\% y República Unida Tanzania con un $13.4 \%$. Sin embargo, es necesario aclarar que estas cifras parten de un reporte realizado por los mismos hombres y que si bien se conservan, el mayor problema es el subregistro, pues aun cuando no se saben cifras exactas, el número de hombres que no reportan el acto pueden conformar un número bastante alto.

Por otro lado, están los trabajos de Elizabeth Kramer (1998), quien, por medio de una detallada historia de las leyes, identifica que la ausencia de los hombres en los estudios y análisis de la violencia sexual ha sido característica, a pesar de que está comprobado que es un problema que es de larga data. Este trabajo es uno de los más completos a la hora de analizar las leyes sobre violencia sexual e identificar la forma en que tanto mujeres como hombres han jugado en determinado papel. Sumado a lo anterior, Kramer introduce un elemento importante en la invisibilización del hombre por parte de los organismos internacionales y por los nacionales, y es el de la víctima. Es decir, cómo se entiende la víctima y cómo es catalogada por estos organismos. Kramer, entonces hace un seguimiento de la evolución del tratamiento hacia el tema de violencia sexual y de la víctima, mostrando cómo la responsabilidad solía y en algunos escenarios suele recaer sobre ella (Kramer, 2006, pp. 295-300).

Ahora bien, el artículo de Patricia Tarre y Salvador Leyva (2015), Violencia sexual contra el hombre: avance jurisprudencial de la Corte Interamericana de Derechos Humanos, puede catalogarse el más claro y detallado, ya que analiza tres momentos históricos en los que la Jurisprudencia de la Corte Interamericana de Derechos Humanos ha tratado este tema. Tarre y Leyva identifican una primera

8 De la violencia sexual contra hombres en cárceles, es necesario resaltar los trabajos realizados en EE.UU., pues esta situación se ha convertido en un problema notorio. Se recomienda los artículos de Donaldson (1993); Sexual victimization in priosions and jails (2008-2009), este último uno de los informes más completos y detallados sobre esta problemática.

9 Se recomienda para el tema de violencia sexual contra hombres, ritos de iniciación y en contextos laborales el texto de S. Axam y Zalenne (1999).

10 Respecto a la violencia sexual en estudiantes universitarios puede consultarse el trabajo de Sasha N. Canan (2016), quien analiza esta problemática sobre todo en EE. UU. 
etapa que comprende de 1988 hasta noviembre 2006, cuando se tenía conocimiento sobre un tipo de violencia en el que se maltrataban zonas corporales, tales como los genitales, pero no se reportaba ninguna clase de alegatos sobre violencia sexual (Tarre y Leyva, 2015, pp. 78 y 79), este es el caso frente a los hallazgos de las comisiones de la verdad, tanto en Guatemala como en Argentina (Tarre y Leyva, 2015, p. 74); un segundo momento, de 2006 al 2014, cuando a pesar de ya reconocerse actos como violencia sexual, no se hace ningún análisis sobre dicho acto, como sí se consideraba para las mujeres (Tarre y Leyva, 2015, p. 81) y un tercer momento, que inició en el 2014 cuando se calificó formalmente un caso de maltrato contra un hombre como violencia sexual (Tarre y Leyva, 2015, p. 85) y ocurre con un caso referido a Colombia, relacionado con los desaparecidos del Palacio de Justicia ${ }^{11}$.

Este trabajo es fundamental dentro de los que se han mencionado, porque es único en analizar casos en Latinoamérica durante las dictaduras y este es uno de los temas que menos se han explorado respecto a estos casos.

Hasta aquí pueden agruparse una serie de trabajos sobre la cuestión de los organismos internacionales y puede determinarse que, en conclusión, estos han descuidado notoriamente el tema de la violencia sexual contra hombres, y que ello ha influido directamente en la poca importancia de esta problemática. Sin embargo, las consecuencias a parte de la poca visibilización, son aún más graves, y es la ausencia de estrategias para impedir y solucionar este tipo de hechos contra los hombres, e incluso fortalecer el discurso en el que como bien lo dice Elysia R. Ruvinsky (2012) en su artículo "My Heart Bleeds, But Where To Take My Grief Is Not There:" Wartime Sexual Violence Against Men in the Balkan and Great Lakes Regions, el problema es la dificultad en relacionar víctima y masculinidad, es decir en otras palabras, la feminización de la victimización de violencia sexual (p. 64). Es así que desde este escenario, se condiciona también al mismo hombre y se hace menos probable una denuncia, de tal manera que se entra en un callejón sin salida que repercute, como ya se ha dicho, en la invisibilización de este tipo de hechos.

\section{Violencia sexual contra hombres. Definición, causas y aportes teóricos}

En cuanto a definiciones, causas y aportes teóricos sobre la violencia sexual, este tema es uno de los más importantes, ya que se adentra en el interés por comprender en detalle qué sucede con los hombres y por qué.

11 La tercera etapa se refiere, por ahora, solamente a la sentencia del caso "Rodríguez Vera y otros ('Desaparecidos del Palacio de Justicia') vs. Colombia” emitida el 14 de noviembre de 2014, en la cual la Corte calificó un maltrato contra un hombre como violencia sexual. En dicho caso, la Corte dio por probado que una de las víctimas, José Vicente Rubiano Galvis, fue detenido y "sometido a múltiples golpes y descargas eléctricas en el estómago y testículos, mientras lo interrogaban y buscaban que confesara ser guerrillero o su colaboración con el M-19". La Corte consideró que dichos maltratos, junto con otros que se dieron por probado, constituyeron tortura (Tarre y Leyva, 2015, p. 86). 
Para empezar, es necesario mencionar a tres académicos que se han dedicado a estudiar este tema con detalle, ellos son, Sandhesh Sivakumaran (2005), Hilmy Zawaty (2006) y Abdullah Khan (2002). Este último, considerado un trabajo muy importante porque proviene desde la criminalística y propone un enfoque y unas definiciones que más adelante utilizarán otros estudios, entre ellos los de Sivakumaran y Aliraza Javaid. También es importante porque es el único que de manera muy detallada aborda el tema de la violencia sexual y su relación con los medios de comunicación, tema del cual se hablará más adelante.

En cuanto a Sivakumaran, este se menciona como uno de los estudiosos del tema más importantes, porque ha desarrollado varias investigaciones sobre la violencia sexual, tanto con postulados teóricos como análisis sobre su protagonismo en organismos internacionales, como se comentó anteriormente (2005, 2007 y 2010). En cuanto a planteamientos teóricos, Sivakumaran (2005, p. 1285) se centra en el análisis de lo que él denomina la mancha de la homosexualidad, primer término fundamental a la hora de abordar la violencia sexual contra hombres y en el caso de Aliraza Javaid (2006), este se dedica a analizar el tema de la masculinidad hegemónica, otra categoría que sin duda debe ser tenida en cuenta en los estudios de este tipo de agresiones.

Ahora bien, Sivakumaran parte del hecho de que este tipo de actos victimizantes no son nuevos, e intenta dar una explicación del porqué este tipo de hechos no son denunciados. Aparte de mencionar aspectos, tales como la vergüenza o la poca atención a este tema, resalta la existencia del problema de la homofobia y como esta se convierte en una mancha para el hombre (2005, pp. 1285-1288), es decir, lo que él denomina la mancha de la homosexualidad.

Partiendo de esta categoría, Sivakumaran encuentra que, por lo general, hay una vinculación directa de la sociedad y sus supuestos, entre el hombre abusado sexualmente y la homosexualidad, relación que incluso la misma víctima hace (Sivakumaran, 2005, p. 1286), razón vital por la cual las denuncias no se dan. Esta relación que él encuentra, hace parte precisamente de la mancha de homosexualidad, porque, aunque él niega rotundamente que quien es violado, lo sea por ser homosexual, la sociedad sí hace este tipo de asociaciones. Incluso la misma víctima las puede llegar a hacer. Este precisamente es el error que según Sivakumaran ha llevado a que este tipo de violencia sea silenciado.

Postulado que es apoyado por varios académicos entre los que es necesario mencionar nuevamente a Michelle Davies y Paul Roger (2006, p. 371) y Stemple (2009), quien, con base en los argumentos de Jeffrey Weeks (1985) asegura que:

Un acercamiento a la violación masculina que asume que todas las víctimas son homosexuales no sirve, pues además de su inexactitud sería perpetuar aún más la posición sesgada que los hombres reales deben ser capaces de evitar su propia violación (p. 633).

Muy ligado a este análisis está el trabajo de Elizabeth Kramer (1998,) quien parte del postulado de la homosexualidad, pero lo concreta en el escenario judicial, donde según ella, el argumento más utilizado para negar este tipo de hechos es 
el de la homosexualidad como causa fundamental. En términos de Kramer, ese sesgo anti-gay parte de dos suposiciones erradas que impiden analizar la problemática. 1. se concibe que los hombres involucrados son gays y 2. Que los violadores tienen un interés sexual, de tal manera que se invisibiliza que este tipo de agresiones son una manifestación de dominio y poder (Kramer, 1998, pp. 311-115).

Es precisamente esta conclusión la que analiza Aliraza Javaid (2016, p. 284), quien se dedica a explicar por qué se da la violencia sexual contra el hombre, y argumenta, igual que la mayoría de los académicos que se citan en el presenta texto, que este es un acto para ejercer poder y control sobre la víctima o sobre una población.

Sin embargo, él introduce una nueva categoría, la de masculinidad hegemónica que, desde su perspectiva, explica por qué se da la violación sexual y porqué se invisibiliza. Aliraza, al igual que quienes recurren a la explicación de poder y masculinidad hegemónica, parten de los postulados de Lees (2002) y Stanko (1990), quienes aseguran que, "los hombres violan a otros hombres como una manera de impulsar, mantener y ejecutar la masculinidad hegemónica, es decir, el macho victimario busca el poder y control sobre sus subordinados como víctima impotente" (traducción propia) (p. 284).

Y Groth y Burgues (1980), quienes además consideran que "La agresión sexual mejora la masculinidad del agresor sexual al eliminar la de sus víctimas” (p. 284).

Este concepto de masculinidad hegemónica agrupa reacciones, tales como la vergüenza, el dolor, la imposibilidad de impedir la situación, lo cual recae sobre los estereotipos de la sociedad en la que hay una idea dominante del ser hombre.

Aliraza tiene dos estudios más denominados, 1. Male Rape: The 'Invisible' Male Why is it that male rape victims are less focused on than their female counterparts by criminology, state agencies, and the media? Y 2, Male Rape: The Unseen World of Male Rape, en el que se dedica a explicar la normalización del abuso sexual contra los hombres y cómo esto se ve reflejado en las respuestas, tanto de la policía como de los medios de comunicación $(2014,2014 b)$.

Después de mencionar a estos académicos, tres de los cuales pueden considerarse pioneros en el tema, vale la pena enunciar otros estudiosos que han enriquecido el análisis con sus aportes.

Entre estos se pueden mencionar a Ruth Graham (2006), Dustin Lewis (2009), Elizabeth Kramer (1998) y Sue Rochman (1991), quienes muy de la mano de Aliraza (2016) consideran que la violencia sexual contra hombres, en vez de ser un impulso sexual del victimario como lo explicaba en tiempos anteriores la criminalística ${ }^{12}$, es una muestra

12 Respecto a la criminología y el problema de la violencia sexual contra hombres, Ruth Graham (2006) encuentra tres etapas claves. La primera se refiere a la criminalística que únicamente se enfocaba en las víctimas mujeres; la segunda etapa es la que se identifica con la masculinidad y se reconocen las diversas masculinidades, y la tercera etapa es la que se caracteriza por recurrir al psicoanálisis. Por otro lado, khan identifica algunos cambios desde la criminología, sobre todo frente a la víctima, es así como evidencia cómo hasta 1970 fue ignorada la violencia sexual contra el hombre. Además de ello, se evidencia cómo el tratamiento a la víctima fue cambiando. Primero con la victimología positivista, la cual le transfería toda la culpa a la víctima. Segundo con la 
de poder y control de dicho victimario frente a la víctima, a su familia o a su comunidad. Con relación a este tema, cabe destacar a Sue Rochman quien además de analizar la problemática de la violencia sexual, se introduce en el estudio del "ser víctima", y argumenta que la invisibilización de hombre como parte afectada de la violencia sexual hace parte de una jerarquización de la víctima en la sociedad $\mathrm{y}$ de un tipo de merecimiento y aceptación social de unas víctimas sobre otras (1991, p. 4).

Muy de la mano de los estudios del "ser víctima" de violencia sexual, están quienes analizan cómo se ha venido analizando y definiendo. Algunos de estos trabajos son los de los psicólogos Michelle Davies y Paul Rogers (2006), quienes hacen una revisión de literatura sobre cómo se consideraba culpable a la víctima de violencia sexual a través de los años, evidencian los diferentes tipos de culpa, entran en diálogo con el feminismo y emiten algunas recomendaciones para solucionar esta tendencia a culpabilizar a la víctima; el trabajo de Thimoty Kdaugherty y Jody A. Esper (1998) y Michelle Davies y Paul Roger (2006, pp. 369-370).

Ahora bien, hay otros estudios que parten de muchos de los postulados anteriores, pero que incluyen en la discusión elementos como la etnia, la religión o tipos de comunidades, los cuales resultan ser influyentes en casos donde la violencia sexual contra el hombre es en sí misma un arma o estrategia propia de la guerra. En este sentido, los estudios de Dubrasvka Zarkov (2001), Elysia Ruvinsky (2012); la investigación de Abu Ghraib (s.f.), Adam Jones y Agusta del Zotto (2002), Hilmi Zawati (2006), Will Store (2011), Miranda Alison (2007) y Wendy Bracewell (2000), Gabrielle Ferrales, Mollie Nyseth Brehm y Sucy Mcelrath (2016) son completamente claros al mencionar cómo en Yugoslavia, en Runda, en Darfur, Irak, en Cosovo, Congo, Uganda y Burundi la lógica de la violencia sexual contra hombres respondía a una expresión de superioridad y dominación suprema de una etnia sobre otra, de un grupo sobre otro o de una religión sobre otra.

Estos estudios, por medio de un análisis detallado de fuentes, encontraron que las cifras de hombres víctimas de violación sexual en escenario de guerra son alarmantes, por ejemplo, en el caso de la ex Yugoslavia, según Zawati, se reportan más de 4000 hombres croatas y más de 50 hombres en Irak.

Casos que a pesar de que son citados en uno que otro documento, no han sido públicos ni mencionados en los medios de comunicación, como puedo evidenciar Zarkov en su tesis, quien después de hacer un análisis de los principales periódicos encontró un gran silencio sobre dichos actos (2011, pp. 69-73).

Estos estudios aparte de tener el valor de conceptualizar el tema de violencia sexual, cuentan con un elemento de gran importancia, el cual se refiere al análisis de este tipo de hechos dentro de un espacio definido, es decir, que sus aportes

victomología radical, la cual inicio una incorporación de todas las víctimas de cualquier tipo, incorporó el enfoque de los derechos humanos y, tercero, la victimología crítica (Khan, 2002, pp. 13-22). 
parten de una identificación y análisis de casos concretos en los escenarios reales de conflicto que les han permitido demostrar la existencia de este tipo de violencia, lo cual constituye un gran aporte para controvertir los estudios que argumentan la poca existencia de análisis sobre violencia sexual contra hombres, debido a los pocos casos que existen y a este argumento, Fred Fuchs (2004) dice: "la ausencia de evidencia respecto a los casos de violencia sexual contra el hombre muestra más el estigma presente en la sociedad para hablar de estos temas que la recurrencia del hecho" (p. 98).

Por otro lado, están los estudios de Cory Freivogel (2010), Dustin Lewis (2009), Katerine Mackinnon (1997), Augusta del Zotto y Adam Jones (2002) y Elisya E. Ruvinsky (2012), quienes analizan la violencia sexual contra hombres, ya no solo desde el concepto de mancha homosexual y de la masculinidad hegemónica, sino desde la feminización del hombre. Para Freivogel, por ejemplo, el único propósito de la violencia sexual contra hombres, por lo menos en la guerra, es la feminización del enemigo por medio de la cual se humilla y debilita al otro (2010, p. 10). Por la misma línea Lewis identifica que en los conflictos, efectivamente se le transfiere al hombre una condición femenina, lo cual es clave para humillar y derrotar al enemigo, pues en la sociedad, la violación es incompatible con la masculinidad (2009, pp. 7-8).

Ahora bien, en un intento por vincular de una forma resumida las categorías que permiten explicar la violencia sexual contra hombres y que cada uno de los estudios que se han citado analizan (masculinidad hegemónica, mancha homosexual, feminización del enemigo, etc.), es fundamental mencionar el trabajo de Gabrielle Ferrales, Hollie Nyseth Brehm y Suzy Mcelrath (2016), quienes a partir de un trabajo que reunió 1136 narraciones de refugiados de Darfur identifican ciertos patrones de la violencia sexual contra hombre adultos y hombres niños.

Este análisis les permite identificar que la violencia contra hombres tiene un efecto de castración por medio de cuatro mecanismos: 1. Homosexualización, 2. Feminización, 3. Daño genital y 4. Asesinato selectivo.

Como se puede ver, las autoras han vinculado tanto los conceptos de homosexualización como los de feminización y masculinidad hegemónica en una explicación general cuyo concepto clave es la castración, la cual se define como toda práctica que disminuye la potencia de los hombres de la familia a la sociedad en general (Ferrales, Nyseth y Mcelrath, 2016, p. 565).

Sumado a esto, este análisis pone en el debate, ya no solo la violencia sexual sino la violencia de género, en la que el hombre debería estar incluido. Es así como se propone que la violencia sexual es solo uno de los tipos de violencia a los que es sometido el hombre debido al género. Este argumento lo muestra cuando trae a colación el tema del asesinato selectivo.

En relación con este último hecho, Charles Carpenter dice que:

Los hombres son mucho más vulnerables a perder la vida. Eso es una gran cosa. Enfermarse, ser violada, son todas cosas muy graves, malas, pero la muerte es muerte y son mucho más vulnerables los hombres que las mujeres a perder la vida (p. 6). 
Además, los hombres son los primeros en ser asesinados en una guerra, en reclutarse, en ponerse presos.

\section{Y Adam Jones asegura:}

El hombre es víctima de reclutamiento forzoso, se les ha negado en ciertos momentos la oportunidad de huir de las zonas de guerra, son encarcelados para evitar su unión o participación en fuerzas de resistencia, ellos a diferencia de los niños y mujeres no entran dentro del tratamiento prioritario (2002).

De la misma manera, explica el informe de ACNUR cuando asegura que tanto las mujeres como los hombres corren riesgo de violencia sexual en momentos de desplazamiento, pues dados sus problemas de subsistencia son más vulnerables a explotación sexual y abuso a cambio de determinados favores.

Cabe aclarar que ninguno de los anteriores estudios intenta negar la importancia ni el trato diferencial de las mujeres, ni desconocer los abusos masivos de los cuales han sido víctimas, pero sí intentan mostrar que el hombre no siempre es el victimario, pues este también vive una violencia de género que se ha cotidianizado y normalizado a través de unos patrones sociales que definen al hombre, como un ser valiente, fuerte y resistente a cualquier agresión.

Adicionalmente, estos tres trabajos, intentan vincular el tema del nacionalismo con la violencia de género y la violencia sexual contra el hombre. Tal vez uno de los grandes aportes de estos trabajos es el valioso intento por visibilizar la violencia de género hacia los hombres, más allá de la violencia sexual e incluir la vinculación directa entre género y nacionalismo, por lo menos en escenarios en que lo étnico cumple un papel fundamental.

Esta vinculación de la violencia sexual contra el hombre y el interés de incluir en la noción de género a los hombres, también la analiza Bennett Capers, quien en su análisis sobre la violencia sexual en escenarios carcelarios asegura que:

Originalmente se ve el género de una manera que inclina la balanza en beneficio de los hombres como padres, esposos, y hombres violadores, y se concibió la violación como únicamente mecanismo de dominación masculina hacia las mujeres (2011, p. 1265).

Con base en todos estos trabajos se puede llegar a la conclusión de que la violencia sexual a nivel general es como bien dice Susan Browmiller destacada feminista:

La violación es un arma de doble filo en la guerra. Está dirigido no solo a la explotación y la humillación de la víctima, sino en la moral de la población en su conjunto. También puede ser un instrumento de venganza por actos atribuidos a los familiares de las víctimas (citado por Jones, 1994, p. 231).

Y a nivel específico el hombre, como dice Bennett Capers la violencia sexual tiene el objetivo de: 
Debilitar a los hombres como protectores de la familia y de la comunidad. Si un hombre es violado sexualmente ¿cómo puede proteger a su familia?

La violencia sexual contra los hombres perpetrados en público puede enviar el mensaje de miedo y la vulnerabilidad de toda la comunidad como la muestra de la impotencia de sus protectores. El desnudo significa exponer y hacer vulnerables a los cuerpos de los hombres, es decir que los autores han eliminado metafóricamente y físicamente la última armadura del hombre soldado (2011, pp. 36-40).

Finalmente, en el tema de las conceptualizaciones y el campo teórico de esta problemática, cabe destacar tres analistas que, a diferencia de la mayoría tuvieron en cuenta una categoría fundamental y que efectivamente ha eclipsado la evidencia de violencia sexual contra el hombre, esta es la tortura.

Tanto Sivakumarán, como Patricia Tarre Moser y Salvador Leyva Morelos; Elysia Ruvnsky y Ellen Anna Philo Gorris hablan de cómo la estrategia de ocultamiento de los hechos de violencia sexual ha sido la categoría de tortura, pues por lo general a esta se hace alusión cuando se trata de analizar los hechos victimizantes del hombre (Philo, 2015, p. 414). Es entonces fácil encontrar narraciones donde se dice que los hombres son torturados en una guerra, mientras las mujeres son torturadas y abusadas sexualmente.

Mientras que con la mujer se da por hecho que existió tortura y se reconoce además la violencia sexual, en el hombre, la categoría de tortura suele incluir todos los actos sean estos sexuales o no. Esto hace que la violencia sexual no se contemple y se pase por alto.

Otro tema que es importante analizar a la hora de referirse a la violencia sexual de los hombres, es el que reseña al hombre como tal, a sus sensaciones, a sus efectos y a sus respuestas fisiológicas. Se pueden encontrar varios estudios que dan cuenta de las consecuencias de la violencia sexual, tanto en el cuerpo como en la salud mental del hombre. Un trabajo que analiza detalladamente esto es el de Sigmund Freud Fuchs (2004), aunque lo enuncian brevemente analistas como Dustin Lewis (2009). Este tema es importante porque es uno de los elementos que producen confusión en la víctima y puede ser un argumento por el cual se soporte la idea de que la culpabilidad de la víctima y fortalecer aquella concepción de la sociedad que se relaciona con la mancha homosexual, lo cual lleva a un escenario en el que no se denuncia el hecho y nuevamente se invisibiliza la problemática que afronta el hombre.

Freud Fuchs analiza en detalle cómo la sociedad concibe al hombre y qué reacción se toma cuando se tiene conocimiento de una víctima masculina. Fuchs, encuentra que socialmente el hombre se considera como sexualmente disponible en todo momento, por lo tanto, las experiencias de violación sexual se vuelven difíciles de creer y un tanto ridículas (2009, p. 94).

Adicional a esta consideración, Freud analiza la relación entre la excitación física y el deseo sexual, con el fin de explicar que, aun cuando el hombre puede tener unas 
respuestas físicas que desembocan en una erección en el momento del ataque, ello no quiere decir que la víctima haya deseado su agresión.

En relación con ello el autor dice que, el tratamiento de una reacción como significante para el consentimiento solo sirve para aislar a los hombres víctimas y perpetúan una forma estereotipada y violenta de masculinidad (Freud, 2004, p. 98).

Así mismo el informe de ACNUR resalta que:

Los hombres no denuncian por el temor que siente el hombre, quien, durante el acto de violencia sexual, en este caso violación sexual experimenta un grado de excitación fisiológica, que en estas circunstancias es una respuesta física del cuerpo, pero ninguna indicación de placer sexual (p. 2).

De igual forma se resalta, por ejemplo, reacciones del hombre, tales como el suicidio y la separación y alejamiento de su familia. Esto como consecuencia de las escasas estrategias diseñadas desde la atención psicosocial con relación a este tipo de hechos (Ruvinsky, 2012, pp. 44- 45).

Según María Emma Wills investigadora del Centro de Memoria Histórica de Colombia,

Cuando violan a un hombre, lo convierten en desechable. En basura. No hay deseo, sino dominación. 'Lo que está en juego es hacerlo sentir inferior y feminizarlo. Después de eso reconstruir la identidad es una tarea titánica', sostiene María Emma Wills, politóloga e investigadora del Centro de Memoria Histórica. Wills reconoce que en Colombia este fenómeno no se ha explorado lo suficiente. Sus investigaciones de campo, eso sí, le permiten inferir que estamos ante un escenario aterrador: 'Hicimos un trabajo sobre violencia sexual en los Montes de María, Magdalena y La Guajira. Tan solo encontramos dos casos de hombres en el Magdalena, pero no hay testimonios directos porque ambos se suicidaron. No pudimos hablar con sus familiares' (Tatiana Escárraga, 6 de septiembre del 2014, El Tiempo).

Para terminar, se debe mencionar el estudio de Nicola Henry (2016), quien llama la atención sobre las distintas maneras para concebir y analizar la violencia sexual. 1. Como forma de violencia interpersonal motivada por individuos; 2 . Como forma de violencia política y, 3. Como engranaje en la economía política y global- acumulación por desposesión. Esta división es importante porque permite diferenciar las formas de analizar la violencia sexual y comprender con detalle cada motivación, contexto y estructura que produce este tipo de hechos de violencia (p. 51).

Finalmente, es importante hacer alusión a los trabajos que proponen una crítica evidente a las teorías o estudios de género y al feminismo en particular. En estos trabajos se pueden destacar Nicola Henry (2016), Ellen Anna Philo (2015) y Noreen Abdullah-Khan (2020), este último considerado uno de los trabajos más completos a propósito de la violencia sexual contra el hombre.

En el caso de Abdullah-Khan, por ejemplo, desde la criminología, hace un estudio sobre la naturaleza e impacto de la violencia sexual en la vida de los hombres, 
por medio de entrevistas, análisis de diarios y encuestas a policías. En este trabajo, además, se analiza este tipo de violencia desde el positivismo, el feminismo y las masculinidades y las diferentes respuestas a través de la historia de la criminología y su concepción de violencia sexual contra los hombres.

Abdullah-Khan (2002), además, se dedica a analizar la violencia en las cárceles, el mito que gira en torno a la homosexualidad, los mitos culturales sobre la violación masculina y algunas leyes sobre esta problemática. Una de las críticas más importantes es la de la tendencia a evidenciar solo la violencia sexual perpetrada a las mujeres y no hacia los hombres, y la tendencia, además, a centrar el análisis en el victimario (impulsos incontrolables, enfermedad mental, pérdida momentánea de control e instigación de la víctima).

De igual forma, analiza la violencia sexual desde el psicoanálisis y la psicología evolutiva (2002, pp. 63-65).

Posteriormente, se dedica a resaltar los aportes y a evidenciar las debilidades del feminismo y los estudios de las masculinidades a propósito de la violencia sexual contra el hombre. Inicia con un breve recorrido sobre las distintas olas del feminismo y muestra cómo han sido visibilizados e invisibilizados los hombres en sus análisis, sin embargo, destaca algunos aportes por parte del feminismo socialista, con el reconocimiento de que las mujeres también cometen actos violentos en torno al género $^{13} \mathrm{y}$ del feminismo radical con el estudio de la violencia sexual y su definición como muestra de poder y control, no obstante, cuestiona la idea de que es el hombre por excelencia el perpetrador de dichos actos (2002, pp. 80-81).

Ahora bien, Abdullah-Khan también resalta elementos que no han sido enunciados y que efectivamente cumplen un papel primordial. Uno de ellos es el que se refiere a los recursos económicos y la visibilización de la violencia sexual contra hombres. Abdullah-Khan encuentra que, en algunos casos, hay un gran temor por visibilizar las afectaciones de los hombres, ya que se considera que esto va a disminuir los recursos destinados para la atención de las mujeres (2002, p. 93), discusión que también debe ser analizada desde el temor de ciertas organizaciones a visibilizar a los hombres y retroceder en el tiempo en cuanto al reconocimiento de la mujer, sin embargo, no hay análisis que se dediquen a estudiar este tipo de preguntas.

Finalmente, Abdullah-Khan concluye que:

Hay una necesidad urgente de desarrollar un modelo adecuado para explicar la violencia masculina contra los hombres y las mujeres con el fin de validar las experiencias del sexo masculino. Mientras que el feminismo surgió como un desafío a las explicaciones biológicas para el crimen también ha sido culpable de excluir a las víctimas masculinas y no darles voz, pues han etiquetado al hombre como autor de violencia y ha aislado de su agenda a las víctimas masculinas (2002, p. 94).

13 El tema de las agresiones a los hombres por parte de las mujeres, consultarse en (Davies y Roger, 2006, p. 372). 
Además, retoma lo planteado por Lees (1997) quien afirma que:

La violación masculina no niega la relación entre violación y dominación masculina (ya sea hombres o mujeres). De hecho, todas las investigaciones sobre la violación masculina han demostrado claramente que la motivación primaria de la violación ha sido la dominación, poder y control (p. 97).

En cuanto a Nicola Henry, ella destaca tres críticas importantes respecto al estudio de la violencia sexual en la guerra, la primera, el esfuerzo por construir la víctima sujeto sin analizar a fondo la cuestión de los autores, las causas estructurales y las condiciones, es decir, que la visión de la víctima es monolítica y universal; la segunda es, que las mujeres han sido siempre consideradas las víctimas y al hombre se le ha descuidado; la tercera, se refiere a que se ha ido creando una jerarquía de las víctimas y, cuarta, se resalta a las mujeres como víctimas pasivas, ocultando el papel de perpetradoras o de resistentes (2016, p. 46).

Igual que los anteriores autores, Henry se dedica a reparar algunos de los enfoques que han estudiado la problemática desde el positivismo o el enfoque cognitivo, y considera que se han olvidado completamente la estructura, por lo tanto, resalta la teoría interseccional, donde según ella, se tienen en cuenta las jerarquías de poder desde los diferentes tipos de discriminación, sean estos raza, sexo y clase (Henry, 2016, p. 51).

Dentro de la crítica a la teoría de género y al feminismo, como se dijo al principio, todos los autores destacan un elemento u otro, sin embargo, la reflexión puede resumirse en que, a pesar de que el feminismo ha visibilizado problemas fundamentales respecto a las problemáticas de la mujer, también ha influido notoriamente en la invisibilización de los problemas de género que afrontan los hombres. Esto quiere decir que ha mantenido una definición del "ser hombre" fuerte, valiente y libre de agresiones que, al parecerse son propias de las mujeres, como es el caso de la violencia sexual.

Por otro lado, las investigaciones de género han reducido su campo de estudio a la mujer, y ello nuevamente ha excluido a los hombres de las problemáticas de género, las cuales no solo responden a la violencia sexual, sino a la tortura, al desplazamiento, al reclutamiento, entre otras.

\section{Respuestas y vías}

Este es otro tema fundamental, pues aparte de visibilizar y analizar la problemática de violencia sexual contra los hombres, es necesario emitir una serie de alternativas que lleven a reconocer este tipo de hechos y brindar soluciones posicionadas en la ley, en la atención humanitaria, en los organismos internacionales y nacionales, en los centros de salud y en la academia.

Las rutas y soluciones que se han definido hasta ahora por los estudiosos del tema, abarcan cuatro escenarios, 1. Los conceptos y bases teóricas sobre la violencia sexual; 2. El campo legal, 3. Organismos internacionales; 4. Los medios de comunicación. 
En cuanto a los conceptos y bases teóricas sobre la violencia sexual, Dustin Lewis (2009) asegura que, "una manera de prevenir este tipo de violencia es el uso de la definición de violencia sexual que incluya la focalización de la violencia imputada, percibida o real de la sexualidad del individuo".

Por otro lado, recomida en términos jurídicos que:

Los redactores de tratados, juristas fiscales y académicos deben hacer conciencia por lo menos en tres cuestiones: 1. Clasificación y definición estereotipadas del Derecho Internacional, el cual ha excluido a los hombres; 2. Deslegitimar algunos estereotipos referentes a la heterosexualidad y homosexualidad de un delito contra la mujer y el hombre y, 3. Ampliación de la definición de la violencia sexual (2009, p. 47).

De igual forma recomienda un sistema de denuncias que les permita la entrada a las víctimas de este tipo de hechos.

Lara Stemple por su parte, es mucho más clara al resaltar la necesidad de crear marcos conceptuales fuera de los derechos de la mujer, de tal manera que la violencia sexual no se reduzca a la violación de las mujeres. Así mismo, considera la importancia del marco legal internacional y sugiere que se contemple el enfoque de violación al hombre a nivel nacional (2006, pp. 640-642).

Elizabeth Kramer (1998) también resalta la importancia de intensificar la protección a los hombres por medio de leyes de protección y estatutos que demuestren que son víctimas de esta agresión lo cual quiere decir que es necesario subsanar ese abandono tanto nacional como internacional (pp. 302-305). Además, hace énfasis en la importancia de aumentar la notificación de la violación, por medio de la creación de vías, de denuncias y metodologías que ayuden a narrar este tipo de hechos y que se anule el temor de las víctimas de ser burladas o de ser catalogadas con una orientación sexual particular (pp. 308-309).

Ahora bien, Elisya Ruvinsky llama la atención sobre la necesidad de cuestionar la idea de tortura frente al hombre, ya que esta muchas veces ha llevado a invisibilizar la problemática de los hombres (2012, p. 81).

Por la misma línea, Benett Capers, sugiere que es muy importante repensar la violencia sexual, es fundamental el análisis de datos provenientes de organismos fiscales y policiales y se debe manejar una neutralidad de género en los procesos de violencia sexual, repensar las sentencias cuyo resultado sea una reforma real que repercuta en la visibilización de este tipo de hechos en los hombres (pp. 1297-1308).

Sin embargo, y como un aporte fundamental, Donaldson afirma que:

Para que los hombres estén cómodos con el uso de estos programas en función de prevenir y tratar la violencia sexual contra ellos, tenemos que hacer el trabajo con la opinión pública. Se necesitan programas dirigidos específicamente a los hombres y estos no existirán hasta que la realidad de la violación masculina se convierta en un hecho de conocimiento público. Sin educación permanecerá enterrada toda la cuestión de la violación de los hombres (citado por Rochman, 1991, p. 89). 
En cuanto a las vías y rutas de solución, debe aclararse que todos los analistas de este tema sugieren elementos para poder visibilizar el tema, sin embargo, el punto de encuentro es la necesidad de ampliar la noción de género y manejar una orientación de género neutra en la que tanto la mujer como el hombre sean analizados de la misma manera, a pesar de los enfoques diferenciales que existen actualmente.

En cuanto a trabajos producidos en Colombia, como se dijo en el inicio del escrito, solo pueden identificarse una tesis de la Universidad de los Andes, dirigida por María Emma Wills, titulada De lo que no se habla: violencia sexual contra los hombres en el marco del conflicto armado. Trabajo que describe algunos estudios sobre la problemática y evidencia algunas situaciones de violencia sexual en el marco del conflicto armado, rastreado por medio de archivo de prensa. El objetivo de este trabajo es explicar por qué ha sido invisibilizado este tema por la academia y evidenciar qué factores impiden contemplar esta problemática. Sin embargo, aunque es un buen avance, carece de cifras y casos que evidencien la existencia de este tipo de violencia.

Finalmente, se debe mencionar que, si bien en el presente artículo se hizo alusión únicamente a trabajos consignados en libros o en artículos académicos, cabe resaltar tres personas que desde el escenario de las ONG han ido trabajando con hombres víctimas de violencia y han realizado diferentes esfuerzos para visibilizar este tipo de hechos. Cris Dolan en Uganda y creador de Refugec Law Project ${ }^{14}$; Alstair Hikton, fundandor de God Men Project ${ }^{15}$ y Ken Clearmater, los tres creadores de South South Institute.

Este Instituto se concentra en ayudar a los hombres víctimas de este tipo de violencia y se encarga por medio de las redes y la web a difundir y visibilizar este tipo de hechos, con el fin de lograr una legislación y una atención que tenga en cuenta a los hombres.

Adicionalmente, se puede encontrar otros proyectos, tales como Genderside Watch, el cual intenta visibilizar el asesinato selectivo, tanto de hombres como mujeres, y en donde se reportan casos en los cuales los hombres han sido víctimas no solo de violencia sexual sino de otro tipo de agresiones por género ${ }^{16}$.

Para finalizar, cabe destacar cómo un informe que es casi el único que se ha hecho en el que se tiene en cuenta de manera detallada la problemática de los hombres, este es el realizado por ACNUR en el 2012, que recrea la situación de los hombres y los niños sobrevivientes de violencia sexual y de género durante el desplazamiento forzado, cuyo objetivo fue resaltar la necesidad de diseñar programas que permitieran incluir a los hombres y los niños víctimas de violencia sexual y diseñar una orientación para lograr la denuncia y la superación de las víctimas.

14 https://refugeelawproject.org/index.php/aboutus/our-profile

15 https://goodmenproject.com/author/alastair-hilton/

16 http://www.gendercide.org/ 
Adicionalmente, proporciona una serie de soluciones e indicadores para la eventual identificación de víctimas de esta violencia ${ }^{17}$ y propone algunas reflexiones importantes para la sociedad en general:

La comunidad entera debe tener en cuenta y ser sensible a los temas relacionados con la violación sexual de género. Se debe entender que las potenciales víctimas incluyen a los hombres. Los hombres y los niños deben saber que las amenazas o los actos de violencia sexual contra ellos son reconocidos y comprendidos. Las mujeres y las niñas necesitan saber que sus familiares hombres y amigos pueden sufrir de violencia sexual de género (Acnur, 2012, p. 10).

\section{CONCLUSIONES}

A lo largo de este balance teórico se ha intentado mostrar varios elementos en torno a la violencia sexual contra el hombre. El artículo intenta, como se ha dicho al inicio, mostrar los principales estudios sobre violencia sexual contra el hombre y la multiplicidad de temas que giran en torno a esta problemática, por lo general muy poco explorada.

Después de revisar cada uno de los trabajos y encontrar sus postulados y argumentos, puede concluirse cuatro elementos fundamentales. El primero se refiere a la poca atención al tema de violencia sexual contra el hombre, con excepción de los libros que se han reseñado en este artículo. Sin embargo, es posible identificar unos intereses crecientes por esta problemática, lo cual se demuestra con el aumento de producción de artículos y por los esfuerzos que algunas personas hacen para atender y visibilizar la problemática.

El segundo elemento, es la necesidad de visibilizar esta problemática por medio de un trabajo de conceptualización que ayude a comprender la violencia sexual y la violencia de género, en especial cuando el hombre es la víctima. De estos trabajos se ha podido ver que la violencia sexual hacia el hombre es más común de lo que se cree, se han podido encontrar reportes que dan cuenta de este tipo de actos en escenarios de guerra, en donde las cifras han sido alarmantes, pero desafortunadamente la denuncia ha sido escasa. Este precisamente es el gran reto que esconde el estudio de la violencia sexual hacia el hombre, es decir, qué rutas diseñar para impulsar la denuncia y qué estrategias de prevención pueden proponerse. Esto va ligado directamente con el cuarto elementos, el cual se refiere a la revisión y reelaboración de conceptos, tales como género y violencia sexual, lo que significa una ampliación de conceptos que les den cabida a los hombres como víctimas, sin desconocer su

17 En cuanto a los indicadores para la identificación de los casos, el informe dice que los sobrevivientes masculinos no suelen denunciar los incidentes inmediatamente, con frecuencia lo hacen cuando los efectos físicos del ataque requieren una intervención urgente. Para atender este tipo de casos es necesario entender las necesidades de los hombres y niños sobrevivientes, realizar un tratamiento médico, un tratamiento para su salud mental y salud social. Es fundamental brindar apoyo para obtener medios de vida sostenibles y brindar protección legal (Acnur, 2012, pp. 6-9). 
rol como victimarios. Aquí es donde se propone una nueva mirada hacia el hombre, ya que este también se encuentra en situaciones de vulnerabilidad y de inferioridad frente a otros hombres; donde su cuerpo también puede hablar de la guerra y de los efectos de ella.

Esto se puede ver y entender en algunos testimonios en los que las narraciones de los hombres demuestran un estado diferente al que por lo general se ha trasmitido cuando se habla del hombre fuerte, viril, valiente e inmune. Sus narraciones por el contrario dejan ver inseguridad, miedo y vulnerabilidad.

Esto también se debe poder asumir desde la sociedad, porque sin duda alguna son víctimas silenciadas cuya implicación es el olvido. El olvido de sus condiciones materiales, de su salud física y mental y de su superación frente a actos de violencia sexual.

Es necesario recordar, como bien lo dicen Lees (2002) y Stanko (1990) y Weiss (2010) que la masculinidad es una construcción social y cultural, y reconocerlo es fundamental para visibilizar este tema.

\section{REFERENCIAS}

Abdullah-Khan, N. (2002). Survivors of male rape: the emergence of a social and legal issue. Center of Criminology.

Acnur. (2002). El trabajo con hombres y niños sobrevivientes de violencia sexual y de género durante el desplazamiento forzado.

https://www.acnur.org/fileadmin/Documentos/BDL/2012/8988.pdf

Aliraza, J. (2015). The dark side of men: the nature of masculinity and its uneasy relationship with male rape. Journal of men Studies, 23(3).

Aliraza, J. (2016). Feminism, masculinity and male rape: bringing male rape "out of the closet". Journal of Gender Studies, 25(3), 283-293.

Alison, M. (2007). Wartime sexual violence: women's human rights and questions of masculinity. Review of International Studies.33, 75.90.

Alizara, J. (2014). Male Rape: The 'Invisible' Male Why is it that male rape victims are less focused on than their female counterparts by criminology, state agencies, and the media? Journal of Criminology.

Bassiouni, M. C. y McCormik, M. (1995). Sexual violence. An invisible wapor of war in the former Yugoslavia. Ocassional paper, No. 1. International Human Rights Law Institute.

Capers, B. (2011). Real Rape Too. California Law Review, 99.

Carpenter, C. (2006). Recognizing Gender-Based Violence Against Civilian Men and Boys in Conflict Situations, Security Dialogue, 37(1), pp. 83-103.

Daugherty, T. (s.f.). Victims characteristics and attributions of blame in male rape. American Psycological Association. (s.d). 
Davies, M. y Rogers, P. (2006). Perceptions of male victims in depicted sexual assaults: A review of the literature. Aggression and Violent Behavior, 11(4), 367-377.

Deborah, Z., y Axam, H. (1999). Simulated sodomy and other forms of heterosexual 'horseplay': same sex sexual harassment, workplace gender hierarchies, and the myth of the gender monolith before and after oncale. Yale Journal of Law \& Feminism, Volume 11, Issue 1.

Del Zotto, A. y Jones, A. (2002, marzo 23 a 27). Maleon-Male Sexual Violence in Wartime: ¿Human Rights’ Last Taboo? trabajo presentado en la Convención Anual de la International Studies Association. Recuparado el 15 de enero de 2010, de http://adamjones.freeservers.com/malerape.htm

Ferrales, G., Nyseth, H. y Mcelrath, S. (2016). Gender-Based Violence Against Men and Boys in Darfur: The Gender-Genocide Nexus. Gender and Society, 30(4).

Freivogel, C. (2005). Feminización del enemigo (s.d).

Freud, S. (2004). Male Sexual Assault: Issues of Arousal and Consent. Law Journal. Cleveland State University.

Graham, R. (2006). Male rape and the careful construction of the male victim. Social \& Legal Studies.

Groth, A.; Burgess, A. W. (1980). 'Male Rape: Offenders and Victims'. American Journal of Psychiatry, 137(7): 806-810.

Henry, N. (2016). Theorizing wartime rape: Deconstructing Gender, Sexuality, and Violence. Gender and Society, 30(1).

Huertas, O., y Amaya, C. (2016). Tríada del derecho penal internacional. Bogotá: Grupo Editorial Ibáñez.

Jones, A. (1994). Gender and Ethnic Conflict in ex-Yugoslavia. Ethnic and Racial Studies, 17(1), 115-134.

Jones, A. (2002). Gender and Genocide. Journal of Genocide Research. 4(1) 65-94.

Kramer, E. (2020, abril). When men are victims: applying rape shield laws to male same-sex rape. New York University Law Review, 95(1).

Lees, S. (1997). Rulling passions: sexual violence reputation and the law Buckingham: Open University press.

Lees, S. (2002). Carnal Knowledge. Rape on Trial. London: The Women's Press Ltd.

Lewis, D.A. (2009). Unrecognized Victims: Sexual Violence Against Men in Conflict Settings Under. International Law Wisconsin Journal of International Law, 27(1), 1-49.

Pérez, J. S. (2011). Historia del feminismo. Madrid: Catarata.

Philo, E. A. (2015). Invisible victims? Where are male victims of conflict-related sexual violence in international law and policy? European Journal of women's Studies, 22(4). 
Rochman, Sue (1991). Silent victims: bringing male rape out of the closet. The Advocate. 582, 38-43.

Russell, W. (2007). Sexual Violence Against Men and Boys. Forced Migration Review (27), 22-23.

Ruvinsky, E. (2012). My heart bleeds, but where to take my grief is not there: 1 Wartime sexual violence against men in the balkan and great lakes regions. Holocaust and Genocide Studies. University of Amsterdam.

Sivakumaran, S. (2005), Male/Male Rape and the "Taint" of Homosexuality. Human Rights Quarterly, 27(4). 1274-1306.

Sivakumaran, S. (2005). Male/Male Rape and the 'Taint' of Homosexuality. Human Rights Quarterly, 27(4), 1274-1306.

Sivakumaran, S. (2007). Sexual Violence Against Men in Armed Conflict. European Journal of International Law, 18(2), 253-276.

Sivakumaran, S. (2010). Del dicho al hecho: la ONU y la violencia sexual contra hombres y niños durante conflictos armados. International Review.

Stanko, E. A. (1990). Everyday Violence: how women and men experience sexual and physical danger. London and San Francisco: Harper Collins.

Stemple, L. (2009). Male Rape and Human Rights. Hastings Law Journal, 60, 605.

Storr, W. (2011). The rape of men: the darkest secret of war. (s.d.).

Tarre, P. y Leyva, S. (s.f.). Violencia sexual contra el hombre: avance jurisprudencial de la Corte Interamericana de Derechos Humanos. México.

Weiss, K. (2010). Male Sexual Victimization Examining Men's Experiences of Rape and Sexual Assault. Men and Masculinities, 12(3).

Zarkov,D. (2001). The body of the other man. Sexual violence and the construction of masculinity, sexuality and ethnicity in Croatian Media. En Caroline, O, M., Moser y Fiona Clarck. Victims, perpetrators or actors? Gender, armed conflict and political violence, USA: Zed Books.

Zawati, H. (2006). Torture: Providing Reparation and Treatment and Preventing Impunity. Alemania. 